\title{
Desarrollo de competencias para la orientación educativa en la formación inicial de profesores
}

\section{Sandra Paola Sunza-Chan}

https://orcid.org/0000-0003-1964-8571

Universidad Autónoma de Yucatán,

México

ssunza@correo.uady.mx

\section{Resumen}

Una de las áreas disciplinares y de práctica que configura el quehacer del educador es la orientación educativa. El objetivo de este trabajo es describir las condiciones metodológicas más relevantes para el desarrollo de competencias para la orientación educativa durante la formación inicial docente. Para tal efecto, se desarrolló una investigación cualitativa, de alcance descriptivo, con estudiantes y profesores de la Licenciatura en Educación de la Universidad Autónoma de Yucatán, México. El método es fenomenológico hermenéutico. Los resultados muestran la relevancia de los proyectos de práctica y el servicio social como ejes fundamentales del desarrollo de las competencias en orientación educativa.

\section{Palabras clave (Fuente: tesauro de la Unesco)}

Enseñanza y formación; desarrollo de competencias; formación de docentes; formación de profesores; México; orientación escolar; Universidad Autónoma de Yucatán.

Recepción: 16/08/2019 | Envío a pares: 23/10/2019 | Aceptación por pares: 05/11/2019 | Aprobación: 06/11/2019 


\title{
Developing Educational Guidance Skills in Initial Teacher Training
}

\begin{abstract}
One of the disciplinary and practice areas that shape the educator's work is educational guidance. This paper intends to describe the most relevant methodological conditions to develop educational guidance skills during initial teacher training. To this end, qualitative research with a descriptive scope was conducted with students and professors of the Education program at the Universidad Autónoma de Yucatán, Mexico. The method used is of a hermeneutical phenomenological type. Results show the significance of practice projects and social service as central concepts in the development of educational guidance skills.
\end{abstract}

\section{Keywords (Source: Unesco Thesaurus)}

Teaching and training; skills development; teacher training; teacher education; Mexico; educational guidance; universidad Autónoma de Yucatán. 


\title{
Desenvolvimento de competências para a orientação educativa na formação inicial de professores
}

\author{
Resumo \\ Uma das áreas disciplinares e de prática que configura o fazer do educador é a orien- \\ tação educativa. O objetivo deste trabalho é descrever as condições metodológicas \\ mais relevantes para desenvolver competências para a orientação educativa duran- \\ te a formação inicial docente. Para isso, foi desenvolvida uma pesquisa qualitativa, \\ de alcance descritivo, com estudantes e professores da Licenciatura em Educação da \\ Universidad Autónoma de Yucatán, México. O método éfenomenológico hermenêu- \\ tico. Os resultados mostram a relevância dos projetos de prática e o serviço social \\ como eixos fundamentais do desenvolvimento das competências em orientação \\ educativa.
}

\section{Palavras-chave (Fonte: Tesauro da Unesco)}

Ensino e treinamento; desenvolvimento de habilidades; orientação escolar; México; formação de professores; Universidad Autónoma de Yucatán. 


\section{Introducción}

En la educación superior, el desarrollo de competencias adquiere un matiz único, debido a su cercanía con el mundo laboral, y enfrenta el reto de responder a las demandas de los diversos contextos productivos. El caso del profesional de la educación no es la excepción, pues este ha sido un campo prolífico de reflexiones teóricas e investigaciones con respecto al desarrollo de competencias para su formación inicial y continua (Mas y Olmos, 2016; Zabalza, 2012; Tejada, 2013; Perrenoud, 2004). De modo más puntual, el desarrollo de competencias para la orientación educativa, como una de las áreas relevantes dentro de los procesos de formación inicial docente, ha sido menos estudiado, debido a que la investigación se ha centrado más en profesionales de la orientación educativa en ejercicio, lo que ha dejado un vacío de investigación, hacia el cual se enfocó este trabajo.

Así, han surgido varias propuestas con respecto a cuál debe ser el perfil de competencias del profesional de la educación para ejercer funciones de orientación educativa, a la luz de las demandas de la sociedad en este campo (CDSGC, 2011; ACLPP, 2008; Maughan, Coyle, McGowan y Wroe, 2016; AIOEP, 2003). Sin embargo, tales estudios no abordan cómo pueden desarrollarse estas competencias en los procesos de formación docente, es decir, se señala el punto de llegada, pero no se reflexiona ni se hacen propuestas puntuales acerca de las condiciones metodológicas a tomar en cuenta en los procesos educativos encaminados a tal fin.

Este tema adquiere un interés aún más sobresaliente si se toman en cuenta las transformaciones educativas actuales, plasmadas en las reformas que se han dado a nivel internacional y en México, las cuales apuntaron hacia la incorporación del enfoque de competencias para la educación en sus diferentes niveles, incluyendo el superior. Precisamente, los resultados de este estudio pretenden profundizar en la comprensión del modo en que un programa educativo centrado en el desarrollo de competencias es llevado a la práctica y a través de qué procesos favorece el desarrollo de los saberes necesarios para un desempeño eficaz en el ámbito de la orientación educativa, desde la perspectiva de los estudiantes y los profesores implicados.

En función de las ideas previamente expuestas, el objetivo de la presente investigación es describir las condiciones metodológicas más relevantes para el desarrollo de competencias en orientación educativa durante la formación inicial docente, desde la perspectiva de estudiantes en fase de egreso y profesores vinculados con estos procesos en un programa de licenciatura perteneciente a una universidad mexicana. Esto cobra una especial importancia si se toma en cuenta que en México las investigaciones realizadas al respecto son insuficientes y que en la Licenciatura en Educación de la Universidad Autónoma de Yucatán, en la que se centra el trabajo, no se han realizado estudios previos.

\section{Antecedentes y fundamentación teórica}

Puede decirse que la formación por competencias ha estado presente en México desde hace más de tres décadas. De acuerdo con el análisis contextual de desarrollo de competencias en México realizado por Monzó (2011), tanto el Instituto Nacional para la Educación para Adultos (INEA) como el Consejo de Normalización y Certificación de Competencia Laboral (Conocer) han tenido un papel relevante en la conceptualización de las competencias en este país y en la estructuración de estrategias para abordarlas en la práctica. También se refiere a otras instituciones que se constituyeron a fin de desarrollar competencias laborales a través de la formación técnica en el nivel medio superior, entre ellas: el Colegio Nacional de Educación Profesional Técnica (Conalep) en 1978 y los Centros de Estudios Científicos y Tecnológicos (Cecyt) en los años 80, los Centros de Estudio Tecnológico Industrial y de Servicios (Cetis), los Centros de Bachillerato Tecnológico Industrial y de Servicios (свтіs), los Centros de Capacitación para el Trabajo Industrial y de Servicios (Cecatis) y los 
Centros de Bachillerato Tecnológico Agropecuario (CBTA), por mencionar algunas. Asimismo, de modo formal, en el año 1993 inició el desarrollo de la Reforma Integral de Educación Básica (RIEB) que modificó el plan de estudios de educación preescolar en 2004, y el de educación primaria y el de educación secundaria entre 2009 y 2011 (SEP, 2002, 2011; Ruiz, 2012). En el caso del bachillerato, la Reforma Integral de Educación Media Superior (Riems) inició en el año 2008 (Diario Oficial de la Federación, 2008).

De acuerdo con Castellanos, Morga y Castellanos (2013), las reformas previamente mencionadas han estado influenciadas en México por los proyectos y las tendencias políticas, económicas y sociales internacionales. De este modo, se hace evidente que la educación basada en competencias va permeando paulatinamente todos los niveles educativos del sistema mexicano. Este cambio ha iniciado desde el ámbito curricular, a través del establecimiento de perfiles de egreso, la identificación y construcción de competencias genéricas y el diseño programas de estudio basados en ese enfoque, ante lo cual resulta indispensable acelerar los procesos de estudio e investigación de esta propuesta, a la luz de las demandas impuestas por las reformas.

En el caso de la educación superior, las transformaciones han sido diversas, al no existir un proceso normativo de reforma como en los niveles educativos ya señalados. Es indispensable mencionar que la incursión de este modelo al ámbito de la educación superior ha tenido características distintivas con respecto a cómo se ha dado en los niveles más básicos. Al respecto, Lasnier (2000), Bunk (1994), Le Boterf (2001, en Navío, 2005) y Martínez, Martínez y Muñoz (2008) coinciden en que las competencias en este nivel deben ser vistas como una combinación compleja de recursos necesarios para ejercer una profesión, que se encuentran estrechamente ligadas a la resolución de problemas y a la acción pertinente en un contexto particular.

De este modo, las competencias vinculan los saberes técnico, metodológico, participativo y perso- nal. Al respecto, es posible señalar varias aportaciones que han abonado a la comprensión del desarrollo de las competencias en este nivel educativo; por ejemplo, Echeverría (2003, en Hernández, Martínez, Da Fonseca y Rubio, 2005) y Martínez et al. (2008) se enfocan en la conceptualización de los saberes profesionales, y Bunk (1994) se centra en la definición de las competencias de acción profesional.

Es así como el desarrollo de competencias en el nivel superior conlleva una importante carga enfocada en la práctica en contextos reales, la cual tiene como base una estrecha relación entre la universidad y la sociedad. En el ámbito de la formación de profesionales de la educación, se requiere una movilización de los saberes propios de esta área en escuelas de diferentes niveles, de modo que el estudiante tenga la oportunidad de actuar en distintos contextos y de enfrentarse con situaciones que integren retos en cada una de las competencias que debe dominar.

Asimismo, Tejada (2013) señala que es indispensable considerar relevante el papel de la formación en la profesionalización del educador, en la cual se requiere una directa conexión con la práctica desde la formación inicial, apoyando planteamientos de modalidad dual, es decir, alternando la universidad con el trabajo en el ámbito educativo. Esta idea es igualmente válida en lo que respecta al desarrollo de competencias para la orientación educativa en la formación inicial del docente y adquiere aún más relevancia si se toma en cuenta la complejidad de esta función, la cual queda clara en la conceptualización de esta disciplina, que puede ser definida como un "proceso de acción continuo, dinámico, integral e integrador, dirigido a todas las personas, en todos los ámbitos, facetas y contextos a lo largo del ciclo vital, y con un carácter fundamentalmente social y educativo" (Martínez y Martínez, 2011, p. 24).

Así, es necesario señalar que los procesos formativos para el desarrollo de competencias en el ámbito de la orientación requieren integrar expe- 
riencias que representen un reto para el alumnado y lo acerquen a situaciones que le permitan practicar la esencia del trabajo orientador, dado que, como señalan Álvarez y Romero (2007), esta función se caracteriza por requerir de profesionales reflexivos que, además de seleccionar y elaborar técnicas adecuadas para cada necesidad, saben aprender de su práctica, partiendo de posicionamientos personales, filosóficos y epistemológicos de la educación.

En este sentido, autores como Aránega (2013) analizan las condiciones que deben reunirse para proveer una formación profesional basada en competencias, así como los retos para el docente implícitos en la inclusión de competencias en la educación superior. En primer lugar, la gestión del currículo y los planes de acción docente deben someterse a una revisión continua y debe primarse el trabajo colaborativo a este respecto. Asimismo, la inclusión de las competencias también requiere: a) materiales, estrategias y recursos innovadores; b) un mayor acercamiento entre la escuela y el mundo laboral; y c) docentes capacitados y actualizados en el uso de las TIC y, en general, de las innovaciones existentes en el campo de la educación.

En resumidas cuentas, es indispensable señalar que es imposible lograr el desarrollo de competencias para la orientación en profesionales de la educación, durante su proceso de formación inicial, con los mismos recursos y estrategias que se han empleado por décadas y que no son acordes a la realidad actual. Por ello se hace necesario indagar sobre las condiciones metodológicas más efectivas para la formación de personas competentes en este campo.

\section{Descripción del contexto}

En México, la formación inicial docente es uno de los ámbitos en los que hay una mayor oferta. Según datos del Instituto Mexicano para la Competitividad (IMCO, 2017), existen por lo menos ocho modalidades en las que pueden ser agrupados estos programas educativos. Predominan aquellos centra- dos en didáctica, pedagogía y currículo, en la enseñanza de asignaturas específicas y en la formación docente para la educación básica en el nivel primario, ocupando esta última modalidad el $4^{\circ}$ lugar a nivel nacional de las carreras con más profesionistas.

También puede señalarse que, aunque existen muchos programas enfocados en la formación docente a nivel licenciatura, son pocos los que consideran dentro de sus áreas la habilitación para el desarrollo de funciones propias de la orientación educativa o la atención a las necesidades específicas de los estudiantes. Asimismo, en el estado Yucatán (en el que se centra esta investigación), son escasos los programas enfocados en la profesionalización de los orientadores educativos. Muestra de esto es que, a partir del análisis de contexto realizados previamente a este estudio, fue posible ubicar únicamente dos programas de posgrado activos en esta área. En el caso particular de la Universidad Autónoma de Yucatán (UADY) y de su Facultad de Educación, en la que se centra este estudio, se ha trabajado durante 33 años en la formación de profesionales de la educación, siendo la orientación educativa uno de los ejes curriculares más importantes.

Por otro lado, en el año 2012, la UADY incorporó a su quehacer el denominado Modelo Educativo para la Formación Integral (MEFI), acción que trajo consigo la actualización de los programas educativos de esta casa de estudio, entre los cuales se encuentra la Licenciatura en Educación. El MEFI se constituyó como el modelo rector del trabajo académico de esta universidad, caracterizándose por girar en torno a principios como la responsabilidad social, la flexibilidad, la innovación, la internacionalización, la educación centrada en el aprendizaje y la educación basada en competencias (UADY, 2013).

A partir de esto, en el año 2014 la UADY aprobó un nuevo plan de estudios para la Licenciatura en Educación, el cual se caracteriza por estar basado en competencias y por una estructura general que considera cuatro áreas formativas, entre las que se encuentran la administración educativa, el currículo, 
la docencia y la orientación educativa. Debido a lo reciente de las modificaciones expuestas, la primera generación finalizó sus estudios en 2018, por lo tanto, es indispensable indagar sobre el modo en que estos cambios están impactando en el perfil competencial para la orientación educativa de sus egresados, así como las condiciones más relevantes a tomar en cuenta en los procesos formativos enfocados en este fin, a partir de las experiencias de alumnos y profesores; de este modo, se sientan las bases para la toma de decisiones respecto a las prácticas que deben continuarse y aquellos aspectos que deben ser fortalecidos.

\section{Método}

El método elegido para la realización de esta investigación es cualitativo, con un alcance descriptivo, enfocado en identificar y analizar las condiciones metodológicas más efectivas para el desarrollo de competencias para la orientación educativa, a través de la perspectiva de alumnos en fase de egreso y profesores de un programa de formación inicial docente recientemente actualizado: la Licenciatura en Educación de la Universidad Autónoma de Yucatán.

Para cumplir con el objetivo señalado, se optó por un diseño de investigación fenomenológico hermenéutico, el cual, de acuerdo con Hernández, Fernández y Baptista (2016), tiene como uno de sus propósitos centrales describir las experiencias de los sujetos con respecto a determinado fenómeno. En este caso, se pretende dar voz a estudiantes y profesores acerca de sus perspectivas sobre los procesos de desarrollo de competencias para la orientación educativa, en función de sus experiencias como agentes activos de un programa de formación inicial docente, cada uno desde el rol que le corresponde. Asimismo, se busca analizar los elementos comunes a través de sus opiniones y vivencias, a fin de señalar las condiciones metodológicas más relevantes a considerar en la formación de profesionales de la educación, específicamente en lo que respecta a las competencias para la orientación educativa.
Los participantes fueron 12 estudiantes en fase de egreso de la Licenciatura en Educación de la UADY, de los cuales 9 son mujeres y 3 son hombres, quienes al momento de la realización de la investigación se encontraban en octavo semestre. Los alumnos fueron seleccionados tomando en cuenta la representatividad de los casos, puesto que se eligieron de acuerdo con los contextos escolares (preescolares, primarias, secundarias, bachilleratos o instituciones de educación superior) en los que desarrollaron su proyecto de práctica, requisito que forma parte de las planeaciones didácticas de las asignaturas de este eje curricular.

Asimismo, participaron los 6 profesores involucrados en los procesos vinculados al campo formativo de orientación educativa perteneciente a este plan de estudios. De estos informantes, 5 son mujeres y 1 es hombre, todos con experiencia profesional en el campo de la formación docente, la orientación educativa y la investigación; sus perfiles profesionales se ubican en el ámbito de la psicología con posgrados en educación (4 docentes) y en el área de educación con posgrados en orientación e inclusión educativa (2 profesores).

La recolección de datos se realizó a partir de entrevistas grupales semiestructuradas, una enfocada en los 12 alumnos participantes y la otra dirigida a los 6 profesores que formaron parte de este estudio. Estas fueron grabadas en audio y transcritas con el apoyo de un procesador de textos; las transcripciones fueron añadidas a una base de datos y se establecieron las unidades de análisis con el apoyo del software ATLAS.TI 8.1.0, tomando como base los objetivos centrales de la investigación. Seguidamente se generaron las categorías y familias a través del análisis de los textos derivados de las narrativas de los participantes en torno al objeto de estudio.

De este modo, se realizó un análisis detallado de las perspectivas de los informantes, tomando en cuenta la naturaleza de sus aportaciones a la luz de su rol como profesores o estudiantes, para identifi- 
car los puntos comunes relativos a las condiciones metodológicas que señalan como relevantes en el desarrollo de competencias para la orientación educativa, las cuales fueron enriquecidas posteriormente con los aportes teóricos derivados de otras investigaciones, a fin de fundamentar su relevancia en los procesos de formación inicial docente.

\section{Resultados y discusión}

Los estudiantes y profesores fueron interrogados sobre distintos aspectos del desarrollo de competencias a partir de los procesos implicados en la formación inicial del docente, a fin de que compartieran su perspectiva, con base en su experiencia en la Licenciatura en Educación de la Universidad Autónoma de Yucatán. Las respuestas proporcionadas fueron transcritas y procesadas con técnicas cualitativas de análisis del discurso, prestándose especial atención a aquellas que permitieran establecer condiciones metodológicas relevantes en la formación de profesionales de la educación competentes para el ejercicio de la orientación educativa en diversos contextos y niveles educativos.

Así, fue posible identificar dos ejes de análisis principales: en primer lugar, las experiencias formativas en sí mismas y, en segundo lugar, el papel de los agentes implicados en el proceso y su contribución al desarrollo de las competencias. Con respecto a las experiencias, se señalan las siguientes categorías: proyectos de práctica ligados a las asignaturas del eje formativo, oferta de cursos optativos, servicio social y prácticas profesionales, experiencias laborales afines al área y realización de tesis. Por otro lado, el segundo eje de análisis giró en torno a categorías ligadas a los agentes involucrados en el proceso formativo y fueron las siguientes: rol de los pares, acción docente, agentes del contexto de práctica y rol del profesor tutor. La integración de familias y categorías derivadas del análisis del discurso de los participantes se presenta en la Figura 1.

\section{Figura 1. Familias y categorias derivadas del análisis cualitativo del discurso de alumnos y profesores, Licenciatura en Educación - UADY}

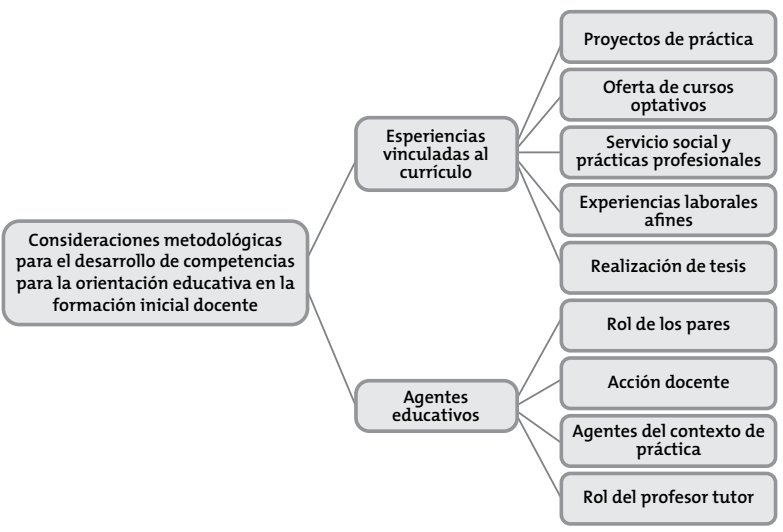

Fuente: elaboración propia.

A continuación, se examinarán los resultados en cada uno de estos ejes, iniciando con las categorías que se mencionaron más entre alumnos y profesores. Para tal efecto, el análisis se basa en algunas de las aportaciones textuales de los informantes, citadas de acuerdo con la codificación asignada (EGP: entrevista grupal a profesores; EGA: entrevista grupal a alumnos), a la cual se añade una letra ( $\mathrm{H}$ : hombre, $M$ : mujer) para identificar el género del participante, y los datos de la fecha de la realización de la entrevista grupal, a fin de facilitar su identificación. Asimismo, se complementarán estos resultados con el análisis de las condiciones metodológicas a tomar en cuenta en la formación de este tipo de profesionales.

\section{Experiencias vinculadas al currículo que contribuyen al desarrollo de competencias para la orientación educativa}

\section{Proyectos de práctica ligados a las asignaturas del eje formativo}

La primera consideración metodológica respecto del desarrollo de competencias para la orientación educativa en los procesos de formación inicial docente refiere a los proyectos de práctica ligados a las asignaturas que forman parte del eje formativo en cuestión, puesto que los resultados del presente 
estudio indican que estas fueron de las experiencias que más contribuyeron al logro de las competencias de los estudiantes. Desde la perspectiva de los alumnos, los requerimientos de los proyectos, la vinculación entre varias asignaturas en ellos y los retos que les planteó los obligaron a regular su propio proceso de aprendizaje y enriquecer sus conocimientos, tal como puede leerse a continuación:

Contribuyó para reforzar los contenidos que estábamos viendo en su momento, pero también para incluir cosas que no estábamos viendo; o sea, de acuerdo al contexto y a las necesidades que estábamos presenciando, tuvimos que investigar acerca de otras necesidades, de otras estrategias, de otros instrumentos, de cosas que quizás se iban a ver más adelante pero que en ese momento necesitábamos, y teníamos que ver la manera de cómo sacarlo adelante [...] lo cual nos dio la oportunidad de seguir enriqueciendo nuestros conocimientos. (EGA-M, 14/06/2018)

En las palabras citadas anteriormente es posible identificar algunos de los beneficios de la puesta en marcha de procesos formativos basados en proyectos y el aprendizaje centrado en problemas, puesto que los estudiantes reconocen que los retos de los proyectos les hicieron indagar más y emprender acciones hacia la superación de los obstáculos. Al respecto, autores como Legault (2012) señalan que se requiere incorporar métodos de pedagogía activa, puesto que resultan más compatibles y coherentes con los modelos de formación por competencias; entre estos, se pueden contar el aprendizaje basado en problemas, los estudios de caso, las simulaciones y juegos de roles, los grupos de discusión, el aprendizaje por proyectos y las prácticas profesionales en contextos reales.

Asimismo, los profesores opinaron que este tipo de proyectos ayudó a los estudiantes al desarrollo de la responsabilidad en la toma de decisiones, así como al desarrollo de las habilidades de gestión. Llama mucho la atención el hecho de que ambos ac- tores (maestros y alumnos) se plantean estas experiencias como oportunidades para que los estudiantes contrasten la teoría contenida en las asignaturas con la realidad práctica de lo que ocurre en los contextos educativos.

En relación con esto, existe aún un debate abierto sobre el equilibrio que debe existir entre la teoría y la práctica en este tipo de procesos. Henao y Martínez (2016) distinguen tres modelos: en primer lugar, el técnico, que aborda la teoría enfocada en la resolución de los problemas más comunes que se pueden encontrar en la práctica; después está el modelo práctico, en el que se busca traspasar los saberes del maestro experto al aprendiz a través de la experiencia y la práctica, perfeccionando el quehacer mediante ensayo y error; por último, es posible señalar el modelo reflexivo, en el que se busca formar a un docente que sea investigador de su propia práctica, por lo cual es necesario exponer al aprendiz a diversas experiencias en las que la sistematización y la certidumbre adquieren un lugar secundario, pues se requiere que esté en contacto con variables aleatorias e inestables, propias de la realidad educativa. Este último modelo es más pertinente a las necesidades de formación de profesionales de la educación en el modelo de competencias y, por ende, al programa educativo al que se hace referencia en este trabajo.

Otro aspecto que considerar fue que ambos actores enfatizaron la importancia del respaldo y la gestión institucional en la implementación de este tipo de estrategias. También se señaló que se requiere que los estudiantes realicen prácticas en diferentes niveles educativos, a fin de evitar una formación fragmentada o con vacíos, de modo que obtengan una visión panorámica de la educación en diferentes realidades.

\section{Oferta de cursos optativos}

Otra de las experiencias formativas más señaladas por los participantes y que debe ser tomada en cuenta como una importante consideración me- 
todológica es la oferta de asignaturas optativas a las que pueden tener acceso los estudiantes como complemento a su formación. Esta afirmación parte de la idea de que es imposible que un currículo abarque todas las áreas disciplinares relevantes para la formación docente, por lo que, en lo referente al ámbito de la orientación educativa, se requiere mantener una oferta de cursos optativos acordes a las necesidades e intereses del alumnado. Los participantes señalaron que deben incorporarse cursos que les permitan la adquisición de competencias en entrevista den orientación y en diseño de instrumentos de evaluación para la orientación educativa, así como aspectos elementales para estructurar adaptaciones curriculares pertinentes a la diversidad presente en el aula.

Por su parte, los profesores concordaron en la percepción de que las asignaturas optativas hacen aportaciones relevantes a la formación de los estudiantes, aunque reconocen que no es posible incluir todo en el plan de estudios, debido a que se incrementaría la carga académica para el estudiante, tal como se señala a continuación: "Otro elemento han sido algunas de las asignaturas optativas que se les han ofertado, que yo creo que varias de esas no deberían ser optativas. Claro que no podemos hacer un programa mucho más cargado, pero hay temáticas que deberían estar para todos, no como opción. Porque después ellos mismos te dicen que tal materia debería ser obligatoria" (EGP-M, 20/07/2018).

Ante esto, se plantea como un importante reto la revisión continua de los saberes esenciales de los planes de estudio, a fin de identificar si existen contenidos obligatorios que deban ser reemplazados por aspectos que se incorporan en las asignaturas optativas y que, desde la perspectiva de los alumnos y profesores, son necesarios para el desarrollo de las competencias fundamentales del perfil del profesional de la educación, en su papel de orientador educativo. De acuerdo con Cepeda (2017), para este tipo de análisis es necesario considerar tanto los rasgos del profesionista que se pretende formar a tra- vés del programa educativo como las necesidades sociales y los cambios concernientes a los saberes científicos que el egresado debe dominar en el ejercicio de su profesión.

\section{Experiencias laborales afines al área}

Otro de los aspectos relevantes que estudiantes y profesores señalaron como una circunstancia favorable para fortalecer el desarrollo de competencias para la orientación educativa fue la posibilidad de que los alumnos pudieran combinar sus actividades académicas con experiencias laborales afines al área formativa; por ejemplo, algunos estudiantes se insertaron en puestos de becarios en diversas áreas de la Facultad de Educación y resaltaron a este respecto los aportes del Departamento de Orientación en la adquisición y fortalecimiento de saberes propios de esta área en los alumnos que ocuparon este cargo.

Entre los principales aportes de esta circunstancia se encuentra lo referente al acercamiento a estudiantes con necesidades específicas de apoyo, la mejor comprensión de la complejidad del trabajo de un orientador y los procesos implicados en esta labor. También los docentes señalaron que este tipo de experiencias apoyaron al desarrollo natural de las competencias propias del perfil del orientador educativo entre los estudiantes que tuvieron la oportunidad de acceder a ellos; no obstante, el impacto abarcó más allá de este ámbito, puesto que también ayudó a que se desarrollen competencias propias de la investigación educativa, como la fundamentación teórica, el análisis crítico, las habilidades de escritura, entre otras. Esto puede corroborarse en la opinión de uno de los profesores investigadores participantes en el estudio:

Yo creo que el Centro de Orientación es un campo muy fértil para poder desarrollar estas habilidades, tanto de orientación como investigativas, porque no solo es la promoción de aplicar instrumentos, sino que el instrumento tiene un sustento: analizate el sustento a la hora de dar el resultado de los instrumentos, haz un reporte, que también 
es otra parte de las competencias de escritura, el comunicarte con colegas. Siento que el Centro de Orientación es un campo muy fértil, como un semillero muy importante para el desarrollo de no solo competencias de orientación, sino también de investigación. (EGP-H, 20/07/2018)

Uno de los aspectos que limitan el impacto de esta experiencia es que se encuentra acotada a un número reducido de estudiantes que pueden acceder a la oportunidad de vivirla, debido a que existen muy pocas plazas. Sin embargo, este resultado apoya la idea de que el desarrollo de competencias para la orientación educativa se ve favorecido si se brindan al estudiante oportunidades laborales planificadas que le permitan probar el nivel de sus saberes en la resolución de problemas específicos, mientras colabora con profesionales en ejercicio que pueden convertirse en modelos de acción profesional, lo cual contribuye a resolver los problemas derivados de la brecha existente entre la universidad y el mundo laboral.

Esta consideración se corrobora las conclusiones de Cuevas e lbarrola (2015) a partir de los trabajos de investigación desarrollados con estudiantes que trabajan, donde se plantea que las experiencias laborales brindan al estudiante oportunidades constantes para construir nuevos saberes y dar paso a la innovación; de este modo, el aprendizaje escolar puede potenciarse si se combina con el desempeño laboral, acompañado con el ejercicio de la metacognición y la práctica reflexiva, como herramientas que abonen al desarrollo de las competencias profesionales.

\section{Servicio social y prácticas profesionales}

El servicio social en México es un requisito obligatorio para los estudiantes que se forman en instituciones públicas de nivel superior, debido a que se plantea como una retribución social y, por ende, se busca que los alumnos se incorporen a proyectos con impacto social y que, a la vez, se encuentren alineados a las características del perfil del programa en el que se forman. Por otro lado, uno de los requisitos de egreso establecidos en el plan de estudios del programa en el que se centró este estudio es la realización de 180 horas de práctica profesional con el objetivo de fortalecer las competencias planteadas en el perfil de egreso y preparar de modo más eficaz a los estudiantes para su incorporación al campo laboral. En ambos casos, el alumno requiere incorporarse a uno de los proyectos aprobados, mismos que emanan de diferentes organizaciones y que son clasificados de acuerdo con el campo formativo en el que se enmarcan las actividades que desempeñará el prestador de servicio social o el practicante, según sea el caso.

En este sentido, los estudiantes insertos en proyectos enfocados en el campo formativo de orientación educativa refieren a esta experiencia como una de las que más contribuyó al desarrollo de sus competencias, tal como lo expresa una alumna: "investigué e hice adaptaciones en el proyecto [...] trabajamos con niños, con profesores, con padres, es decir comenzamos un proyecto desde cero" (EGA-M, 14/06/2018). Otro estudiante señala:

Tuve que investigar, hacer modificaciones, tuve que hacer informes, reportes, estar tratando con niños con discapacidad, darles pláticas; fue algo motivador para mi porque logré algo que jamás hubiera imaginado y que no pensé lograr. No estamos preparados para ciertos contextos, sin embargo, tenemos que investigar, capacitarnos a nosotros mismos para poder solventar estas necesidades, y fue muy bonito, porque tuve la oportunidad de hacerlo. (EGA-H, 14/O6/2018)

Así, puede señalarse que los retos de estas experiencias permiten al estudiante prepararse en áreas específicas del quehacer del profesional de la educación, incluso más allá de los contenidos integrados en el plan de estudios. Ante esto, se puede afirmar que una de las condiciones metodológicas más fuertes para las instituciones que pretenden formar este tipo de competencias es mantener la vinculación con instituciones externas en las que, a partir de proyectos, los estudiantes puedan insertarse en su realidad, identificar necesidades y diseñar, implementar 
y evaluar estrategias en el ámbito de la orientación educativa, de modo que visualicen las implicaciones al respecto, desde la etapa de estudiantes.

En ese sentido, estudios como el de Aguirre, Rodríguez y Caso (2016) muestran que existe un amplio espectro de aprendizajes que pueden adquirirse a través del servicio social, tanto los de nivel disciplinar como las competencias de carácter genérico y transversal. Para esto, plantean lo indispensable de proveer condiciones mínimas, como el apoyo por el cuerpo docente, la gestión de recursos económicos, espaciales y temporales, así como la vinculación al currículo, a fin de potenciar su impacto social y su contribución a la responsabilidad social universitaria y a la justicia social. Por su parte, López, Cedeño, Lescay, Mestre y Meza (2016) señalan que las prácticas profesionales ayudan a reconfigurar el papel de la universidad e incrementar la pertinencia de sus acciones, acercándola a la realidad y actuando como un instrumento de beneficio compartido entre las instituciones de educación superior y los sectores social y productivo.

\section{Realización de tesis como forma de titulación}

Entre las modalidades a través de las cuales puede titularse un estudiante del programa educativo en el cual se centra la presente investigación están el examen general de egreso (EGEL) y la elaboración de una tesis. Con respecto a esta última, estudios como el de Calvo (2009) reportan resultados que permiten visualizar la titulación universitaria como un proceso a través del cual es posible generar conocimiento, por medio de la investigación.

En el presente estudio, los resultados apuntaron a que la realización de tesis es uno de los aspectos que más contribuyen al desarrollo de competencias para la orientación educativa, específicamente en aquellos trabajos cuyo objeto de estudio se encontraba ligado a este campo formativo. Esto puede notarse en el siguiente comentario: "otro elemento serían las tesis; los que realizaron tesis y que están en el área de orientación desarrollaron mucho esto, esa sensibilización hacia el área, ese pensar en el otro y las competencias establecidas, y pudieron vincular mucho de lo que se vivió con el proyecto de investigación concretamente" (EGP-M, 20/07/2018).

Esto va de la mano con la perspectiva de Velásquez (2017), para quien la formación de competencias genéricas puede beneficiarse del desarrollo de investigaciones en las que los estudiantes puedan aplicar los aprendizajes obtenidos a lo largo de su formación, a la vez que continúan su especialización en determinadas áreas vinculadas con su perfil profesional y se fortalece el desarrollo de una mentalidad científica orientada a dar respuestas y proponer soluciones a diversas problemáticas.

\section{Agentes educativos involucrados en el desarrollo de competencias para la orientación educativa}

El segundo eje de análisis, como se mencionó previamente, agrupa a las categorías enfocadas en los agentes involucrados en las experiencias que favorecieron el desarrollo de competencias. A continuación se presenta un análisis de cada una. Nuevamente, los resultados se han organizado en función de aquellas categorías más mencionadas entre los participantes de las entrevistas grupales.

\section{Rol de los pares}

Una de las características del currículo del programa educativo que se analiza es el énfasis en el trabajo colaborativo, el cual tiene un peso muy fuerte en los procesos formativos, puesto que se enfatiza en el desarrollo de habilidades para el trabajo en equipo, de modo que se movilicen actitudes positivas y habilidades interpersonales como la negociación, la comunicación, la asertividad, entre otras.

En lo que concierne al campo formativo de orientación educativa, una gran parte de las actividades de aprendizaje presenciales y no presenciales son llevadas a cabo en grupos; de modo más específico, en la fase práctica, el estudiante debe desa- 
rrollar un proyecto en campo real trabajando en un equipo colaborativo permanente durante dos años consecutivos. Esto explica que el rol de los pares se vuelva tan significativo para ellos en el proceso de desarrollo de sus competencias. Esto puede leerse en el siguiente comentario:

Obviamente los compañeros [son esenciales en el aprendizaje], porque creo que ya casi casi vivías con ellos, de hecho, no solo en el sentido de trabajar con ellos, sino que te ibas a la comunidad con ellos, pasabas hambre con ellos, de todo hacias, y nos tocó ser esas figuras de apoyo en situaciones que estaban pasado por el trabajo, escolar o personal; entonces yo creo que son los agentes más significativos que nos ayudaron a desarrollar nuestras habilidades o a practicarlas. (EGA-M, 14/06/2018)

Resaltan en este sentido los procesos de acompañamiento brindados entre pares, lo que les permitió constituirse en soporte unos de otros; asimismo, se originó de modo natural la práctica de la coevaluación, que ayuda en el autoconocimiento y en la gestión del aprendizaje, lo cual potenció la motivación:

Siempre trabajamos en equipos. Entonces a mí me tocó igual un buen grupo, buenos equipos donde podian decirme "es que necesitas desarrollar esta estrategia, eres buena en esto, pero te falta tal cosa o sigue adelante"; esto me parece buena idea, si lo estas desarrollando de manera correcta. Entonces esta manera de estarnos evaluando y estarnos complementando a mí me ayudó demasiado para reconocer tanto mis fortalezas como mis áreas de mejora. (EGAM, 14/06/2018)

Es necesario recalcar que el trabajo colaborativo resulta un reto para el alumnado, pues implica un continuo aprendizaje mutuo, así como un ejercicio constante de contrastar opiniones propias con las de otros, negociar acuerdos beneficiosos para el colectivo, trabajar para el logro de objetivos comunes, compromiso con el equipo y el proyecto, entre otros. Al respecto, Fombona, Iglesias y Lozano (2016) señalan que las dificultades más importantes están vinculadas con actitudes y conductas de los miembros del grupo, pues este tipo de prácticas puede ser terreno de cultivo para la falta de responsabilidad y compromiso de algunos estudiantes, lo cual impide que el trabajo realizado sea afectivo.

En este mismo sentido, Sempere, García, Marco y De la Sen (2011) concuerdan en que el trabajo colaborativo ayuda a superar las limitaciones de modelos anteriores y fomenta la calidad del aprendizaje, siempre y cuando en su implementación se considere un cambio en los roles tradicionales de maestros y alumnos. De parte del profesorado, este tipo de trabajo plantea serias necesidades, como adoptar el rol de mediador en aquellos casos en que los alumnos tengan posturas encontradas que no puedan resolver por sí solos; asimismo, plantea la necesidad de transformar los mecanismos tradicionales de supervisión, medición y evaluación de los aprendizajes, a fin de responder a las problemáticas que se generan de estas interacciones.

\section{Acción docente}

Continuando con la línea de análisis planteada en el apartado anterior, autores como Jarauta (2014) y Podestá (2014) consideran que el profesorado requiere trabajar en la creación de ambientes estimulantes para el aprendizaje, movilizando relaciones auténticas en los grupos. Para esto, será indispensable que el maestro se aleje de los modelos tradicionales y se posicione como un mediador, orientador y compañero del alumno, actuando como un agente fundamental en la construcción de las competencias.

De modo congruente con esto, entre los agentes cuya acción profesional resultó más relevante en el desarrollo de competencias para la orientación educativa en los estudiantes en fase de egreso de la Licenciatura en Educación (LE) de la UADY se encuentran los profesores. Al respecto, los propios maestros resaltaron que el perfil del profesorado, sobre todo en su experiencia profesional y sus com- 
petencias para la investigación, así como el trabajo en equipo del cuerpo docente fueron clave en la estructuración de los procesos formativos, tal como se lee en el siguiente comentario: "creo que la experiencia que tiene la planta docente en el área, donde el trabajo profesional y de investigación ayuda a la planificación de experiencias, y esa comunión en la planta docente ayuda mucho en la planeación de lo que se va a hacer; ya cada docente le pone su estilo, pero el ponernos de acuerdo es una fortaleza" (EGP-M, 20/07/2018).

En este sentido, Podestá (2014) coincide en que un docente requiere tener experiencia personal en el trabajo colaborativo para ser competente en enseñar a sus estudiantes sobre esta compleja práctica. De modo similar, Antúnez (1999) expresa que los educadores requieren estar coordinados mediante el trabajo colaborativo para contribuir a la educación de calidad; es decir, se necesita que la planta docente dé ejemplo de trabajo colaborativo a través de una práctica que se centre en el trabajo común, la coordinación y la ayuda mutua.

Otro de los resultados relevantes de la presente investigación mostró que los alumnos resaltaron que pudieron ver en sus profesores modelos de las competencias a desarrollar; asimismo, señalaron como otro aspecto favorable la disposición de la planta docente para compartir sus conocimientos y sus experiencias a fin de ayudarles en sus procesos de aprendizaje. Esto puede corroborarse en las siguientes ideas expresadas por uno de los alumnos que participó en el estudio:

A mi me tocó la dicha de tener buenos profesores con los que si me pude reflejar, o sea, al ver al profesor y decir "desarrolla esta competencia en esta forma", y me puedo reflejar en ellos y decir "bueno, ya sé cómo desarrollar esa competencia", y también que compartan sus conocimientos y experiencias, el decir "pues yo también estuve en este momento, en esta situación; entonces te ayudo y te comparto mi experiencia para que tú puedas tener una idea de cómo sobrellevar esta situación". Entonces considero que mis profesores si me ayudaron para poder desarrollar estas competencias. (EGA-H, 14/06/2018)

Autores como Salcedo (2012) manifiestan que la acción docente debe servir de modelo a los estudiantes en cuanto a la actuación estratégica, lo cual solo es posible si se convierte en un ejemplo de las competencias que pretende formar. Este reto implica un alto nivel de desarrollo profesional, puesto que, desde la perspectiva de González (2004), si este no ha alcanzado un alto nivel de madurez profesional, expresado en su motivación por esta profesión, dedicación al trabajo, nivel de experto en su área disciplinar y formación psicopedagógica idónea, será muy difícil que pueda ser considerado un modelo de actuación por el alumnado. A más de esto, Serna, Sánchez y Rubio (2015) señalan que es una necesidad que los docentes cuenten con las competencias básicas en el ámbito afectivo, de modo que sean capaces no solo de enseñar conocimientos, sino también de modelar valores y prácticas éticas en sus alumnos, desarrollando de este modo una nueva competencia profesional.

A partir de esto, es relevante estimular la reflexión sobre el impacto del docente como un modelo en el desarrollo de competencias y sobre la necesidad de una transformación del rol del profesorado, de modo que la distancia entre maestro y alumno se acorte y convierta así el aprendizaje en un proceso más participativo y bilateral. Es importante señalar que existe amplia literatura que documenta los retos que implica para los docentes el trabajo centrado en el desarrollo de competencias del alumnado; por ejemplo, los trabajos de Zabala y Arnau (2007), Villarroel y Bruna (2014), Legault (2012) y Ortega (2010) profundizan en las exigencias didácticas, pedagógicas y evaluativas que este tipo de enfoque acarrea al profesorado.

También se plantea como una necesidad el fortalecimiento del trabajo colaborativo entre el profe- 
sorado, a fin de emprender proyectos que continúen impactando la formación del alumnado. Por último, surgen interrogantes en cuanto a la capacitación docente con miras a incrementar sus competencias para el acompañamiento académico y emocional del alumnado, de modo que se convierta en un guía que, con base en la confianza y el compañerismo, favorezca la motivación y la implicación del alumno en el logro de los objetivos del programa educativo.

\section{Agentes del contexto de práctica}

De modo menos frecuente, pero no por ello menos importante, se identificó que los agentes de los contextos en los que los estudiantes llevaron a cabo sus prácticas influyeron en el desarrollo de sus competencias para la orientación educativa. A este respecto, resaltaron dos puntos relevantes: por un lado, se señala la apertura de las escuelas receptoras de los practicantes, al brindarles la oportunidad de incorporarse a la dinámica organizacional del centro (EGP, M, 20/07/2018), y por el otro, en esta línea, se refiere la posibilidad de estar en contacto con profesionales en ejercicio "con perfiles afines a la orientación, como orientadores o tutores" (EGA-M, 14/06/2018).

La importancia de estas experiencias radica en sus aportaciones a la formación integral del alumnado y en que coadyuvan en la interrelación entre teoría y práctica, a la vez que les ayuda a movilizar saberes que les permitan enfrentarse al complejo y competitivo mundo laboral de la actualidad (Mareque y De Prada, 2018). Asimismo, de acuerdo con Molina, Bolívar, Burgos y Ponce (2007) el practicum representa un contacto reflexivo con el contexto educativo y una oportunidad real para acceder al mundo del trabajo, lo cual coincide con la experiencia de la Licenciatura en Educación de la UADY, donde los estudios de los egresados corroboran que las prácticas en contextos reales son una de las fuentes principales de empleo para los estudiantes que finalizan sus estudios.

\section{Rol del profesor tutor}

Otro agente cuyo papel destacó en el proceso de desarrollo de competencias para la orientación fue el profesor tutor. Al respecto cabe señalar que, entre los servicios de atención al alumnado, la UADY cuenta con un programa consolidado de tutoría individualizada, misma que se concibe como un proceso de acompañamiento al estudiante. Para tal efecto, a cada alumno se le asigna un profesor tutor que da seguimiento a su trayectoria académica desde el ingreso hasta el egreso.

Algunos de los participantes coincidieron en que la figura del tutor fue relevante debido a la experiencia de estos profesionales y a que fungían como modelos de las características del orientador educativo, lo que permitía que los alumnos identificaran las habilidades para la orientación en la práctica de las funciones tutoriales, tal como se enuncia en el siguiente comentario respecto de la tutora: "fue una persona que, cada vez que tenía mis sesiones de tutoría, veía siempre en ella las características de un orientador educativo y, a través de eso, igual aprendía mucho de su experiencia. También me compartía siempre cómo fue cuando ella se estaba formando, [de modo que] yo me veía reflejado en ella y en esas competencias" (EGA-H, 14/06/2018).

Así, es posible ver que la tutoría tiene un alcance que va más allá de sus objetivos formalmente establecidos, puesto que eventualmente puede convertirse en una fuente de interacciones que, de modo indirecto, apoyan la formación del alumnado en lo que respecta a las competencias para la orientación educativa. Este resultado coincide con lo expresado por Pámies y Galindo (2015) tras evaluar la implementación de un sistema de tutorías, en el que encontraron un alto potencial en cuanto a su contribución al desarrollo de competencias transversales. Del mismo modo, Obaya y Vargas (2014) opinan que la tutoría ayuda al desarrollo de competencias de vida, pues aporta a que los alumnos den sentido a sus aprendizajes. 
A manera de cierre de este apartado, es indispensable enfatizar en la complejidad de las experiencias y los agentes que han contribuido a los procesos de desarrollo de competencias para la orientación educativa en los estudiantes en fase de egreso de la LE de la UADY. Puede notarse que, aunque todos están vinculados de algún modo a los planteamientos curriculares, hay hallazgos que muestran que los procesos formativos en la práctica son mucho más complejos que lo que puede estar formalmente estipulado en un plan de estudios. Por esta razón, investigaciones como la que se describe en este trabajo son necesarias y deben ser más frecuentes, a fin de comprender con mayor profundidad las implicaciones, los alcances, los aciertos y desaciertos de los procesos de formación inicial del profesorado.

\section{Conclusiones y recomendaciones}

En respuesta al objetivo central de este estudio de describir las condiciones metodológicas más relevantes en cuanto al desarrollo de competencias para la orientación educativa durante la formación inicial docente, desde la perspectiva de estudiantes en fase de egreso y de profesores vinculados con estos procesos en un programa de licenciatura perteneciente a una universidad mexicana, es necesario señalar, a manera de conclusión, que en la Licenciatura en Educación de la Universidad Autónoma de Yucatán (UADY), las experiencias más relevantes fueron los proyectos de práctica, los cursos optativos, el servicio social, las prácticas profesionales, las experiencias laborales afines al área y la realización de tesis como modalidad de titulación.

En este punto resulta interesante señalar que las condiciones metodológicas apuntan tanto a experiencias planificadas y documentadas en el currículo formal como a vivencias que rebasan lo que es posible prever en los procesos formativos. Muchos de los elementos significativos en los resultados de esta investigación emergen del contacto de los estudiantes con los contextos de práctica pedagógica y profesional, es decir, que se ubican totalmente fuera de las aulas de clase, con lo cual se corrobora la complejidad implícita en la pretensión de contribuir al desarrollo de competencias en un ámbito tan particular como es la orientación educativa. Además de estas experiencias en sí mismas, los participantes señalaron que los agentes educativos implicados en estos procesos guardan mucha importancia; entre los que tuvieron un mayor impacto en el desarrollo de competencias fueron los pares, los docentes, los agentes de los contextos en los que se realizaron las prácticas y los profesores tutores.

Estos resultados arrojan luz para entender qué elementos metodológicos deben ser considerados al momento de estructurar experiencias formativas dentro de los programas de formación inicial del profesorado en las competencias para la orientación educativa, la cual demanda cada vez más la incorporación de profesionales capaces de exhibir un desempeño eficaz ante las complejas demandas sociales propias de nuestro tiempo y en los diversos niveles educativos en los que se enmarca su labor. Al respecto, se coincide con las ideas de Biggs y Tang (2011), quienes afirman que es indispensable cuidar la coherencia entre varios elementos, a saber: las competencias que se pretende desarrollar, los métodos empleados para tal efecto y los sistemas de evaluación que se apliquen, lo cual se puede llamar alineamiento del aprendizaje.

Asimismo, estos hallazgos guardan relación con los planteamientos del Enfoque Socioformativo Complejo (ESC) en lo que respecta a las condiciones pedagógicas necesarias para desarrollar competencias -cuyos principios fundamentales, de acuerdo con Tobón (2012), se centran en la unidad e inclusión en la escuela, a fin de que se favorezca la autorrealización del alumnado a través de un proyecto ético de vida-y en relación con el trabajo colaborativo entre los agentes de la comunidad escolar y de la sociedad en general y su implicación en el desarrollo de las competencias como una vía de transformación social.

Por otro lado, se requiere también un cambio de perspectiva, en el que las problemáticas del en- 
torno educativo circundante sean vistas como oportunidades de aprendizaje, a través de las capacidades de innovación y emprendimiento; asimismo, se ha de fortalecer la práctica reflexiva en las instituciones formadoras de profesionales de la educación, como base de una acción transformadora que sea capaz de adaptar sus procesos a las demandas de aprendizaje de sus estudiantes y a las necesidades sociales emergentes.

Es indispensable señalar que, a partir de los resultados expuestos en este trabajo, se integran ideas que sirven como líneas guías para el desarrollo de propuestas de mejora en la formación de competencias para la orientación educativa de los estudiantes. Esta proposición tendrá impacto en los futuros procesos de actualización y desarrollo curricular del programa educativo en el que se centra. De igual forma, esta investigación aporta ideas que pueden servir como base para guiar innovaciones en lo que respecta a la formación de competencias para la orientación en otros programas educativos.

Entre las recomendaciones derivadas de esta investigación, es posible enfatizar en la necesidad de realizar estudios de evaluación curricular continua en los programas que pretenden formar profesores con base en el modelo de competencias, a fin de identificar las fortalezas y posibles áreas de mejora en este sentido, buscando potenciar los resultados de aprendizaje logrados por el alumnado. Ante esto, resulta un reto vincular a todos los agentes que participan en los programas educativos en las evaluaciones, con miras a ampliar los resultados y obtener un panorama más completo del objeto de estudio.

Por otro lado, surge como una necesidad encontrar los mecanismos idóneos para reestructurar los procesos formativos a partir de los resultados obtenidos en las investigaciones, de modo que se traduzcan en prácticas pedagógicas, académicas y administrativas que favorezcan el aprendizaje del alumnado, respondiendo al modelo de competencias. Para esto, es indispensable el trabajo colaborativo, tanto del personal interno de la universidad (profesores, investigadores, personal administrativo y autoridades) como de aquellos que provengan de los contextos que forman parte del campo laboral del profesional de la educación que pretende egresar.

En resumen, en el desarrollo de competencias como finalidad de la educación no se trata únicamente de un cambio didáctico, sino de una transformación del modo de concebir el hecho educativo. Esto remite a las preguntas fundamentales con respecto a qué competencias deben ser desarrolladas y para qué se deben promover tales aprendizajes.

\section{Referencias}

Álvarez, V.y Romero, S. (2007). Formación basada en competencias para los profesionales de la orientación. Educación XX1, 10(1), 15-37. Recuperado de http://www.redaly c.org/pdf/706/70601003.pdf

Aguirre, L., Rodríguez, J. y Caso, J. (2016). Potencial de aprendizajes en el servicio social universitario. Revista Internacional de Aprendizaje en la Educación Superior, 3(1), 1-12. Recuperado de https://journals.epistemopolis. org/index.php/edusuperior/article/view/503/

Aioep - Asociación Internacional de Orientación Educativa y Profesional (2003). Competencias internacionales para los orientadores educativos y profesionales. Asamblea General, Berna, 4 de septiembre de 2003. 
Antúnez, S. (1999). El trabajo en equipo de los profesores y profesoras: factor de calidad, necesidad y problema. El papel de los directivos escolares. Educar, 24, 89-110. Recuperado de https://ddd.uab.cat/pub/educar/o2 11819Xn24/o211819Xn24p89.pdf

Aránega, S. (2013). De la detección de las necesidades de formación pedagógica a la elaboración de un plan de formación en la universidad. Barcelona: Octaedro.

ACLPP - Asociación Castellano Leonesa de Psicología y Pedagogía (2008). Los servicios de orientación en el ámbito educativo no universitario de Castilla y León.

Biggs, J. y Tang, C. (2011). Teaching for quality learning at university. Berkshire: Open University Press.

Bunk, G. (1994). La transmisión de las competencias en la formación y perfeccionamiento profesionales de la RFA. Revista Europea de Formación Profesional, 1(1), 8-14.

Calvo, M. (2009). La elaboración de la tesis de licenciatura como espacio para la formación y la construcción social del conocimiento. Perfiles Educativos, 37(124), 22-41. Recuperado de https://www.redaly c.org/ pdf/132/13211178003.pdf

Castellanos, N., Morga, L. y Castellanos, A. (2013). Educación por competencias. Hacia la excelencia en la formación superior. México: Red Tercer Milenio.

CDSGC - Committee of Directors of Studies in Guidance Counselling (2011). Counselling Competencies for Guidance Counselling Education Programmes: Interim Guidelines. Recuperado de http://www.thecdi.net/write/Guidance_Counselling_Core_Competencies_Professional_Practice.pdf

Cepeda, J. (2017). Metodología del diseño curricular basado en competencias profesionales. Revista Cientifica Epistemia, 1, 1-10. Recuperado de http://revistas.uss.edu.pe/index.php/EPT/article/view/573

Cuevas, J. y De Ibarrola, M. (2015). Aprender en la simultaneidad: La perspectiva de los estudiantes que trabajan sobre los saberes y competencias que construyen. Revista Mexicana de Investigación Educativa, 20(67), 1157-1186. Recuperado de http://www.scielo.org.mx/scielo.php?script=sci_arttext\&pid=S1405$66662015000400007 \& \operatorname{lng}=$ es\&tlng=es

Diario Oficial de la Federación (2008). Acuerdo número 442 por el que se establece el Sistema Nacional de Bachillerato en un marco de diversidad. Recuperado de http://cosdac.sems.gob.mx/portal/index.php/riems

Fombona, J., Iglesias, M. y Lozano, I. (2016). El trabajo colaborativo en la educación superior: una competencia profesional para los futuros docentes. Educação \& Sociedade, 37(135), 519-538. DOI: https://doi.org/10.159o/ ES0101-73302016147914

González, V. (2004). El profesorado universitario: su concepción y formación como modelo de actuación ética y profesional. Revista Iberoamericana de Educación, 34(1), 1-11. DOI: https://doi.org/10.35362/rie3412934 
ISSN 0123-1294 | e-ISSN 2027-5358 | Educ.Educ. Vol. 22. No.3 | Septiembre-diciembre de 2019 | pp. 448-468.

Universidad de La Sabana | Facultad de Educación

Henao, D. y Martínez, L. (2016). Concepciones de la práctica pedagógica en los diferentes niveles de formación del profesorado. Tecné, Episteme y Didaxis, núm. extr., 725-731. Recuperado de https://revistas.pedagogica. edu.co/index.php/TED/article/view/4662/

Hernández, R., Fernández, C.y Baptista, P. (2016). Metodología de la investigación. 6 ed. México: McGraw-Hill.

Hernández, F., Martínez, P., Da Fonseca, P.y Rubio, M. (2005). Aprendizaje, competencias y rendimiento en Educación Superior. Madrid: La Muralla.

IMCO - Instituto Mexicano para la Competitividad (2017). Compara carreras 2016. Recuperado de http://imco.org. $\mathrm{mx} /$ comparacarreras/\#!/?utm_source=www.comparacarreras.org.mx\&utm_medium =redirects\&utm_ campaign $=301$

Jarauta, B. (2014). El aprendizaje colaborativo en la universidad: referentes y práctica. Revista de Docencia Universitaria, 12(4), 281-302. DOI: https://doi.org/10.4995/redu.2014.5624

Lasnier, F. (2000). Reiussir la formation par compeitences. Montreal: Gueìrin.

Le Boterf, G. (2001). Ingeniería de las competencias. España: Gestión 2000.

Legault, A. (2012). ¿Una enseñanza universitaria basada en competencias? ¿Por qué? ¿Cómo? Revista Electrónica de Desarrollo de Competencias, 5(9).

López, R., Cedeño, G., Lescay, D., Mestre, U.y Meza, H. (2016). Relevancia del proceso de vinculación con la sociedad en la formación de profesionales en instituciones de educación superior del Ecuador. Órbita Pedagógica, 3(2), 61-82. Recuperado de http://www.revista.isced-hbo.ed.ao/rop/index.php/ROP/article/view/70

Mareque, M. y De Prada, E. (2018). Evaluación de las competencias profesionales a través de las prácticas externas: incidencia de la creatividad. Revista de Investigación Educativa, 36(1), 203-219. DOI: https://doi. org/10.6018/rie.36.1.275651

Martínez, P. y Martínez, M. (2011). La orientación en el siglo XXI. Revista Electrónica Interuniversitaria de Formación del Profesorado, 14(1), 253-265. Recuperado de http://www.redaly c.org/articulo.oa?id=217017192020

Martínez, P., Martínez, M. y Muñoz, J. (2008). Aprendizaje de competencias en educación superior. Revista Galego-Portuguesa de Psicoloxía e Educación, 16(1), 1-16. Recuperado de http://ruc.udc.es/dspace/bitstream/ handle/2183/7607/RGP_16_art_13.pdf? sequence=1

Mas, O. y Olmos, J. (2016). El profesor universitario en el Espacio Europeo de Educación Superior. La autopercepción de sus competencias docentes actuales y orientaciones para su formación pedagógica. RMIE, 21(69), 437-470. Recuperado de http://www.redaly c.org/pdf/140/14045395005.pdf

Maughan, M., Coyle, B., McGowan, P. J. y Wroe, P. (2016). Guidance Counselling Core Competencies \& Professional Practice. Ireland: Institute of Guidance Counsellors. Recuperado de http://www.thecdi.net/write/Guidance_Counselling_Core_Competencies_Professional_Practice.pdf 
Molina, E., Bolivar, A., Burgos, A. y Ponce, C. (2007). Mejorar el practicum de Pedagogía. Aportaciones desde la investigación. Revista de Investigación Educativa, 25(2), 443-461. Recuperado de http://www.redaly c.org/ articulo.oa?id=283321923011

Monzó, R. (2011). Formación basada en competencias. El caso de los estudios de la Escuela de Administración de Instituciones, de la Universidad Panamericana en México. Tesis doctoral, Universidad Internacional de Catalunya, Barcelona. Recuperado de http://www.tdx.cat/bitstream/handle/10803/79177/Tesi_Rosa_ Monz\%F3.pdf;jsessionid=41AAFoF78CF5B30A986E76D1AD8FD383? sequence=1

Navío, A. (2005). Propuestas conceptuales en torno a la competencia profesional. Revista de Educación, 337, 214234. Recuperado de http://www.educacionyfp.gob.es/revista-de-educacion/numeros-revista-educacion/ numeros-anteriores/2005/re337/re337-11.html

Obaya, A. y Vargas, Y. (2014). La tutoría en la educación superior. Educación Química, 25(4), 478-487. Recuperado en 12 de julio de 2019, de http://www.scielo.org.mx/scielo.php?script=sci_arttext\&pid=S0187-893X20140 o0400012\&lng=es\&tlng=es

Ortega, M. (2010). Competencias emergentes del docente ante las demandas del Espacio Europeo de Educación Superior. Revista Española de Educación Comparada, 1(16), 305-327. Recuperado de http://revistas.uned.es/ index.php/REEC/article/view/7534

Pámies, M. y Galindo, C. (2015). Tutoría como herramienta académica del desarrollo de competencias transversales. Revista Iberoamericana de Educación, 68(1), 165-178. Recuperado de https://rieoei.org/historico/ deloslectores/6987.pdf

Perrenoud, P. (2004). Diez nuevas competencias para enseñar. Education, 23, 223-229. Recuperado de http:// revistas.um.es/educatio/article/view/127/111

Podestá, P. (2014). El trabajo colaborativo entre docentes: experiencias en la Especialización Docente Superior en Educación y TIC. Congreso Iberoamericano de Ciencia, Tecnología, Innovación y Educación, Buenos Aires, Argentina. Recuperado de https://www.oei.es/historico/congres02014/memoriactei/374.pdf

Ruiz, G. (2012). La Reforma Integral de la Educación Básica en México (RIEB) en la educación primaria: desafíos para la formación docente. Revista Electrónica Interuniversitaria de Formación del Profesorado, 15(1), 51-60. Recuperado de http://aufop.com/aufop/uploaded_files/articulos/1335398629.pdf

Salcedo, F. (2012).Papel del profesor en la enseñanza de estrategias de aprendizaje.Revista Didasc@lia:D\&E,3(3), 17-28. Recuperado de https://dialnet.unirioja.es/descarga/articulo/4230og8.pdf

SEP - Secretaría de Educación Pública (2002). Documento base de la Reforma Integral de Educación Secundaria. México. Recuperado de https://coleccion.siaeducacion.org/sites/default/files/files/reformas_secundaria.pdf

SEP - Secretaría de Educación Pública (2011). Plan de estudios 2011, Educación Básica. México. 
ISSN 0123-1294 | e-ISSN 2027-5358 | Educ.Educ. Vol. 22. No.3 | Septiembre-diciembre de 2019 | pp. 448-468.

Universidad de La Sabana | Facultad de Educación

Sempere, J., García, M., Marco de la Calle, F. y De la Sen, M. (2011). Aprendizaje colaborativo: un reto para el profesor en el nuevo contexto educativo. En El trabajo colaborativo como indicador de calidad del espacio europeo de educación superior (pp. 89-103). Alicante: Marfil.

Serna, O., Sánchez, C. y Rubio, I. (2015). Los nuevos retos y demandas de la educación "desarrollo de competencias". Atenas, 3(31), 1-7. Recuperado de https://www.redaly c.org/pdf/4780/478047207001.pdf

Tejada, J. (2013). Profesionalización docente en la universidad: implicaciones desde la formación. Revista de Universidad y Sociedad del Conocimiento, 10(1), 170-184. DOI: https://doi.org/10.7238/rusc.v10i1.1471

Tobón, S. (2012). El Enfoque Socioformativo y las competencias: ejes claves para transformar la educación. En Experiencia y aplicación de las competencias en la educación y el mundo organizacional. Durango: Redie. Recuperado de http://iunaes.mx/wp-content/uploads/2013/o4/Experiencias-de-Aplicacion.pdf\#page=10

UADY - Universidad Autónoma de Yucatán (2013). Modelo educativo para la formación integral. Documento institucional. Recuperado de http://www.dgda.uady.mx/media/docs/mefi_dgda.pdf

Velásquez, P. (2017). Percepción de la formación universitaria basada en competencias genéricas y capacidades orientadas a la elaboración de tesis en egresados de administración. Tesis de grado, Universidad de San Martín de Porres, Lima. Recuperado de http://www.repositorioacademico.usmp.edu.pe/bitstream/ usmp/2901/1/velasquez_tpa.pdf

Villarroel, V. y Bruna, D. (2014). Reflexiones en torno a las competencias genéricas en educación superior: un desafío pendiente. Psicoperspectivas, 13(1), 23-34. Recuperado de https://www.psicoperspectivas.cl/index. $\mathrm{php} / \mathrm{psicoperspectivas/article/view/335}$

Zabala, A. y Arnau, L. (2007). 11 ideas clave. Cómo aprender y enseñar competencias. Barcelona: Graó.

Zabalza, M. (2012). El estudio de las "buenas prácticas" docentes en la enseñanza universitaria. Revista de Docencia Universitaria, 10(1), 17-42. DOI: https://doi.org/10.4995/redu.2012.6120 